\title{
Cytogenetic Findings on 11,451 Cases of Amniocentesis in Hanoi, Vietnam
}

\author{
Cuong Danh Tran ${ }^{1}$, Van Bich Nguyen ${ }^{1}$, Minh Xuan Thi Nguyen ${ }^{2}$, Lan Ngoc Thi Hoang ${ }^{1}$, Anh Toan Ngo ${ }^{2}$, Toan \\ Van $\mathrm{Ngo}^{1}$, Chau $\mathrm{Ngo}^{3}$, Hue Thi Mai ${ }^{4} \&$ Tung Thanh $\mathrm{Tran}^{3}$ \\ ${ }^{1}$ Hanoi Medical University, Hanoi, Vietnam \\ ${ }^{2}$ National hospital of Obstetrics and Gyneacology, Hanoi, Vietnam \\ ${ }^{3}$ Institute for Global Health Innovations, Duy Tan University, Da Nang, Vietnam \\ ${ }^{4}$ Institute for Preventive Medicine and Public Health, Hanoi Medical University, Hanoi, Vietnam
}

Correspondence: Cuong Danh Tran, Hanoi Medical University, Hanoi, Vietnam. E-mail: trandanhcuong.pstw@gmail.com

Received: April 9, 2018 Accepted: May 2, 2018 Online Published: May 16, 2018

doi:10.5539/gjhs.v10n6p152

URL: https://doi.org/10.5539/gjhs.v10n6p152

\begin{abstract}
Since the 1960s, amniocentesis has become a routine procedure performed in prenatal diagnostic clinics. This study aims to depict the results of amniocentesis, the frequency of chromosomal abnormality and emphasize on amniocentesis indications. A retrospective study was conducted on 11,451 cases who were referred to the Prenatal Diagnosis Center, the National Hospital of Obstetrics and Gynecology, Hanoi, Vietnam from 2012 to 2016. The rate of chromosomal abnormality was $6.7 \%$. The chromosomal aberration in the group with mother or father carrying balanced parental translocations accounted for the highest rate of $22.0 \%$. Triploidy was $1.2 \%$, autosomal chromosome aberration was $59.7 \%$, sex chromosome was $8.3 \%$, and structural rearrangements was $30.8 \%$. Trisomy 21 was the most frequent disorder founded in abnormal ultrasound findings (47.4\%), following by the advanced maternal age (44.1\%). The large sample size of this study provided reliable evidence to support the development of prenatal counseling and pregnancy management programs.
\end{abstract}

Keywords: prenatal diagnosis, karyotype, amniocentesis indications

\section{Introduction}

Since the 1960s, amniocentesis (AS) has become a popular invasive test for those at high risks of genetic diseases (Jacobson \& Barter, 1967; Wang \& Cheng, 2009). Presently, amniocentesis is considered as a routine procedure at many prenatal diagnosis centers aiming to detect numeric and structural chromosomal disorders based on clinical findings and family history (Caron, Tihy, \& Dallaire, 1999; Hook, Cross, \& Schreinemachers, 1983). Pregnant women considered for AS are often of advanced age (over 34 years old) or under 34 in combination with positive maternal serum screening test at first or second trimester (Ferguson-Smith \& Yates, 1984; Wang \& Cheng, 2009). Many previously published studies have proposed other indications such as a study by Caron et al. on the Canadian population for 20 years looking at frequency of chromosomal aberations (Caron et al., 1999) and a study by Yi-Wen et al. in Taiwan for 30 years (Chang et al., 2012). The risk of chromosomal disorders was found to be higher in pregnant women with previous abnormal births, abnormal ultrasound findings, intra-uterine growth restriction (IUGR), or intra-uterine fetal death (IUFD). Tseng et al. demonstrated that the risk of chromosomal abnormalities was higher in the group with ultrasonographic malformations (Tseng et al., 2006). This study consists of 11,451 amniocentesis cases in the National Hospital of Obstetrics and Gynecology- the top-referral hospital in Hanoi, Vietnam from 2012 to 2016 in order to evaluate the various routine indications. The results of this study could hold valuable indications for genetic counseling.

\section{Method}

Clinical records were collected from amniocentesis database from the Center for prenatal diagnosis at the National Hospital of Obstetrics and Gynecology in Hanoi, Vietnam from 2012 to 2016. The indications of amniocentesis for cytogenetic analysis included: (1) previous abnormal chromosome birth, (2) previous congenital malformation births, (3) the history of miscarriage/stillbirth (more than 2 times), (4) parental carriers of chromosomal disorders, (5) positive maternal serum screening tests (cutoff with Down syndrome of 1/320), (6) advanced maternal age 
(over 35 years old), and (7) abnormal ultrasound findings.

Two culture disks $4 \mathrm{ml}$ Amniomax were prepared for each patient (Gibco, ThermoFisher Scientific). Then, the culture media was left in the disks for 10-13 days until the colonies were sufficient (about 30-40 colonies). Routine G-banding was performed for all specimens (Seabright, 1971).

Amniocytes were cultured in the genetic laboratory for about 2 weeks, then harvested for processing of cytogenetic results. Chromosomal analysis was performed in Carl-Zeiss automated microscopy system. All chromosomal disorders were classified into following categories: (1) autosomal chromosome aneuploidies, (2) sex chromosomal aneuploidies, (3) structural rearrangements (reciprocal translocation, Robertsonian translocation, deletion, inversion...). Frequencies of all observed disorders were calculated according to all indications.

\subsection{Ethical Approval}

This study was approved by the Ethical Committee of National Hospital of Obstetrics and Gynecology. Participants were informed of the study purpose, and were asked to give a written informed consent to confirm their participation. Participants could withdraw anytime and their information was kept confidential.

\section{Results}

Table 1. Case number and the rate of chromosomal disorders

\begin{tabular}{llll}
\hline Year & Number of cases & Abnormal number & Frequency of abnormality \\
\hline 2012 & 1447 & 87 & 6.0 \\
2013 & 1830 & 91 & 5.0 \\
2014 & 2701 & 151 & 5.6 \\
2015 & 2558 & 209 & 8.2 \\
2016 & 2915 & 232 & 8.0 \\
Total & 11451 & 770 & 6.7 \\
\hline
\end{tabular}

The number of cases of amniocentesis indicated an increase from 1,447 cases in 2012 to 2,915 cases in 2016. In general, the number of chromosomal disorders was 770 cases (6.7\%), ranging from $5.0 \%$ in 2013 to the peak of $8.2 \%$ in 2015 (Table 1).

Table 2. The prevalence of chromosomal aberrations by indication

\begin{tabular}{lllll}
\hline Indications & $\mathbf{N}$ & $\mathbf{\%}$ & N (Abnormalities) & \% (Abnormalities) \\
\hline Previous abnormal chromosome births & 403 & 3.5 & 14 & 3.5 \\
Previous congenital malformation birth & 859 & 7.5 & 33 & 3.8 \\
History of miscarriage/stillbirth & 2392 & 20.9 & 171 & 7.1 \\
Parental carriers of chromosomal disorders & 123 & 1.1 & 27 & 22.0 \\
Positive maternal serum screening tests & 2354 & 20.6 & 107 & 4.5 \\
Advanced maternal age & 2419 & 21.1 & 68 & 2.8 \\
Abnormal ultrasound findings & 2901 & 25.3 & 350 & 12.1 \\
Total & 11451 & 100.0 & 770 & 6.7 \\
\hline
\end{tabular}

Table 2 illustrates the prevalence of chromosomal aberrations of participants. The most common indication for AS was abnormal ultrasound findings (25.3\%), followed by advanced maternal age $(21.1 \%)$, history of miscarriage/stillbirth (20.9\%), and positive maternal serum screening test (20.6\%). Regarding chromosomal aberrations, the biggest proportion of chromosomal aberrations was found in parental carriers of chromosomal disorders $(22.0 \%)$. 
Table 3. Frequencies and types of chromosomal disorders by indications

\begin{tabular}{|c|c|c|c|c|c|c|c|c|}
\hline & $\begin{array}{l}\text { Previous } \\
\text { abnormal } \\
\text { chromosome } \\
\text { s disorders } \\
\text { N (\%) }\end{array}$ & $\begin{array}{l}\text { Previous } \\
\text { congenital } \\
\text { malformation } \\
\text { birth } \\
\mathrm{N}(\%)\end{array}$ & $\begin{array}{l}\text { History of } \\
\text { miscarriag } \\
\text { e/stillbirth } \\
\text { N (\%) }\end{array}$ & $\begin{array}{l}\text { Parental } \\
\text { carriers of } \\
\text { chromosoma } \\
\text { I disorders } \\
\text { N (\%) }\end{array}$ & $\begin{array}{l}\text { Positive } \\
\text { maternal } \\
\text { serum } \\
\text { screening } \\
\text { test } \\
\text { N (\%) }\end{array}$ & $\begin{array}{l}\text { Advanced } \\
\text { maternal } \\
\text { age } \\
\text { N (\%) }\end{array}$ & $\begin{array}{l}\text { Abnormal } \\
\text { ultrasound } \\
\text { findings } \\
\text { N (\%) }\end{array}$ & $\begin{array}{l}\text { Total } \\
\text { N (\%) }\end{array}$ \\
\hline Triploidy & 0 & 0 & $2(1,2)$ & 0 & $2(1,9)$ & 0 & $5(1,4)$ & $9(1,2)$ \\
\hline \multicolumn{8}{|c|}{ Autosomal chromosomes disorders } & $460(59.7)$ \\
\hline Trisomy 21 & $5(35.7)$ & $13(39.4)$ & $66(38.6)$ & $3(11.1)$ & $30(28.0)$ & $30(44.1)$ & $166(47.4)$ & $313(40.6)$ \\
\hline Trisomy 18 & 0 & $2(6.1)$ & $27(15.8)$ & 0 & $3(2.8)$ & $1(1.5)$ & $73(20.9)$ & $106(13.8)$ \\
\hline Trisomy 13 & 0 & 0 & $4(2.3)$ & 0 & $1(0.9)$ & 0 & $11(3.1)$ & $16(2.1)$ \\
\hline $\begin{array}{l}\text { Autosomal } \\
\text { chromosome } \\
\text { mosaicism }\end{array}$ & 0 & $3(9.1)$ & $4(2.3)$ & 0 & $1(0.9)$ & $2(2.9)$ & $7(2.0)$ & $17(2.2)$ \\
\hline Other & 0 & $1(3.0)$ & $4(2.3)$ & 0 & 0 & 0 & $3(0.9)$ & $8(1.0)$ \\
\hline \multicolumn{8}{|c|}{ Sex chromosomal disorders } & $64(8.3)$ \\
\hline $45, \mathrm{X}$ & 0 & $1(3.0)$ & $2(1.2)$ & 0 & $2(1.9)$ & $1(1.5)$ & $9(2.6)$ & $15(1.9)$ \\
\hline $47, \mathrm{XXY}$ & 0 & 0 & $3(1.8)$ & $1(3.7)$ & $3(2.8)$ & $4(5.9)$ & $8(2.3)$ & $19(2.5)$ \\
\hline $47, \mathrm{XXX}$ & 0 & 0 & $2(1.2)$ & 0 & 0 & $1(1.5)$ & $4(1.1)$ & $7(0.9)$ \\
\hline $47, \mathrm{XYY}$ & 0 & $1(3.0)$ & $4(2.3)$ & 0 & $2(1.9)$ & 0 & $4(1.1)$ & $11(1.4)$ \\
\hline $\begin{array}{l}\text { Sex } \\
\text { chromosome } \\
\text { mosaicism }\end{array}$ & 0 & 0 & $3(1.8)$ & 0 & $3(2.8)$ & $2(2.9)$ & $2(0.6)$ & $10(1.3)$ \\
\hline Other & 0 & 0 & $1(0.6)$ & 0 & 0 & 0 & $1(0.3)$ & $2(0.3)$ \\
\hline \multicolumn{8}{|c|}{ Structural rearrangements } & 237 (30.8) \\
\hline Reciprocal & $2(14.3)$ & $4(12.1)$ & $6(3.5)$ & $9(33.3)$ & $12(11.2)$ & $1(1.5)$ & $7(2.0)$ & $41(5.3)$ \\
\hline Robertsonian & 0 & 0 & $3(1.8)$ & $1(3.7)$ & $5(4.7)$ & $1(1.5)$ & $4(1.1)$ & $14(1.8)$ \\
\hline Inversion & 0 & $4(12.1)$ & $12(7.0)$ & $4(14.8)$ & $14(13.1)$ & $7(10.3)$ & $15(4.3)$ & $56(7.3)$ \\
\hline Deletion & $5(35.7)$ & $3(9.1)$ & $6(3.5)$ & 7 (25.9) & $4(3.7)$ & $2(2.9)$ & $8(2.3)$ & $35(4.5)$ \\
\hline Duplication & $2(14.3)$ & $1(3.0)$ & $7(4.1)$ & $2(7.4)$ & $4(3.7)$ & $4(5.9)$ & $4(1.1)$ & $24(3.1)$ \\
\hline Insertion & 0 & 0 & $3(1.8)$ & 0 & $3(2.8)$ & $1(1.5)$ & $4(1.1)$ & $11(1.4)$ \\
\hline Marker & 0 & 0 & $1(0.6)$ & 0 & 0 & 0 & $1(0.3)$ & $2(0.3)$ \\
\hline Polymorphism & 0 & 0 & $11(6.4)$ & 0 & $18(16,8)$ & $11(16.2)$ & $14(4.0)$ & $54(7.0)$ \\
\hline Total & $14(100)$ & $33(100)$ & $171(100)$ & $27(100)$ & $107(100)$ & $68(100)$ & $350(100)$ & 770 (100) \\
\hline
\end{tabular}

Of the 11,451 cases, $93.3 \%$ had normal karyotype (10,681 cases) and $6.7 \%$ had abnormalities ( 770 cases). In the group of chromosomal aberrations, 9 cases had triploidy (1.2\%), 460 cases had autosomal chromosomes abnormality (59.7\%), 64 cases had sex chromosomes aberrations (8.3\%), and 237 cases had structural rearrangements $(30.8 \%)$.

Triploidy (1.2\%) was found more frequently in pregnant women with abnormal ultrasound findings (5 out of 9 cases), most common images found by ultrasound were enlarged, cystic placenta, and fetal structural anomalies (Table 3). The majority of numerical chromosome disorders was autosomal chromosomal trisomy $(59.7 \%$, 460/770). Trisomy 21 was the most common abnormality among all cases (40.6\%), in which $47.4 \%$ was founded in abnormal ultrasound findings, and $44.1 \%$ was found in advanced maternal age. Trisomy 18 and 13 were found in 106 cases (13.8\%) and 16 cases $(2.1 \%)$, respectively. Additionally, 8 cases of rare aneuploidies were recorded in 
this study: 1 case of trisomy 1, 2 cases of trisomy 22,1 case of trisomy 8,1 case of trisomy 9,1 case of trisomy 12 , 1 case of trisomy 9 , and 1 case of monosomy 10 . In cases of sex chromosome aneuploidies, monosomy $\mathrm{X}$ found in 15 cases $(1.8 \%), 19$ cases were 47,XXY (2.5\%), 11 cases were $47, \mathrm{XYY}(1.4 \%), 10$ cases were mosaicism $(1.3 \%)$, 7 cases were $47, \mathrm{XXX}(0.9 \%)$. Total structural rearrangements were 237 cases $(30.8 \%)$. Inversion was the most frequent aberration detected with chromosome structure (7.3\%), followed by polymorphism $(7.0 \%)$ and reciprocal translocation (5.3\%). Most of the structural rearrangement cases found in abnormal ultrasound findings and history of miscarriage/stillbirth.

\section{Discussion}

The number of amniocentesis cases and chromosomal disorders were gradually increased each year, ranging from $5.0 \%$ in 2013 to the peak of $8.2 \%$ in 2015 . The overall rate of chromosomal disorders was $6.7 \%$. While this result was lower than the result from a study in Turkey conducted on 13,466 amniocentesis cases (Özer, Ünsal, Ayvaz, Şen, \& Baltac1, 2016), it was higher than those of other studies which ranged from $2.7 \%$ to $3.6 \%$ (Chang et al., 2012; Han et al., 2008). Presumably, during the Vietnam War, prior generations were exposed to the large amount of toxic chemicals (Field \& Kerr, 1988), this may lead to the likelihood of chromosomal defects in the offspring. Additionally, as the Prenatal Diagnosis Center at the National Hospital of Obstetrics and Gynecology in Vietnam is the top-referral for prenatal diagnosis, this may explain the high number of high-risk cases in comparison with other facilities.

AS indications based on abnormal findings during prenatal examinations included: (1) previous abnormal chromosome disorders, (2) previous congenital malformation birth, (3) history of miscarriage/stillbirth, (4) parental carriers of chromosomal disorders, (5) positive maternal serum screening tests, (6) advanced maternal age, (7) abnormal ultrasound findings. Amniocentesis involves certain risks for pregnant women such amniorrhea (the absence of menstrual periods), miscarriage, fetal death. Thus, indications for amniocentesis must be under consideration of both risks and benefits.

Abnormal ultrasound finding was the most common amniocentesis indication which accounted for $25.3 \%$ and the rate of chromosomal abnormalities in this group was $12.1 \%$. Previous studies revealed the various rates of chromosomal abnormalities ranging from $4 \%$ to $15.5 \%$ (Chang et al., 2012; Ocak, Ozlu, Yazicioglu, Ozyurt, \& Aygun, 2014; Özer et al., 2016). Although $21.1 \%$ participants were in advanced maternal age, only $2.8 \%$ found with chromosomal disorders. It is suggested that the risk of chromosomal nondisjunction is correlated with maternal age (Hassold \& Hunt, 2009). This was consistent with a large report in Europe with 52,965 pregnant women (Ferguson-Smith \& Yates, 1984). In this study, 123 couples (1.1\%) were carriers for chromosomal disorders, mostly reciprocal translocation and the rate of abnormal offspring this pregnancy peaked at $22.0 \%$. A previously published study found $1.5 \%$ couples with chromosomal aberrations and $11.51 \%$ with chromosomal disorders (Chang et al., 2012). Furthermore, Zepney Ocak et al. (2014) found that $100 \%$ offspring had chromosomal rearrangement among parental translocation carriers (Ocak et al., 2014).

In our present database, couples with previous miscarriage and/or stillbirth were accounted for $20.9 \%$ and the rate of chromosome abnormalities in this group was $7.1 \%$. This figure was even higher than couples with the history of abnormal chromosome disorders and congenital malformation (3.5\% and 3.8\%, respectively). In developing countries, miscarriage and/or stillbirth are highly prevalent due to the poverty and the lack of education (Aminu et al., 2014). In addition, in Vietnamese culture, sexual health topics are often avoided and not up for discussion (Pham et al., 2012). As a result, there is a potential gap in sexual and reproductive health knowledge among Vietnamese women. Thus, it is important to raise the knowledge and awareness regarding sexual and reproductive health among Vietnamese women. We recommend that couples with recurrent miscarriage and/or stillbirth should consider screening for indicated karyotype carriers.

Types of chromosomal abnormalities included triploidy $(1.2 \%, 9 / 770)$, autosomal chromosome disorders $(59.7 \%$, 460/770), sex chromosome disorders $(8.3 \%, 64 / 770)$, structural rearrangements $(30.8 \%, 237 / 770)$ which concurred with previous studies (Chang et al., 2012; Han et al., 2008; Ocak et al., 2014). Trisomy 21 was most frequent, found in $40.6 \%$ of the total abnormal cytogenetic findings; and among those, $47.4 \%$ was with morphological malformations found by ultrasound. The frequency of trisomy 21 in the second trimester was higher because another small proportion of trisomy 21 had been lost spontaneously in utero before delivery (Hook, 1983). Zhang et al recorded that the frequency of trisomy 21 was about $35.6 \%$ of all chromosomal detected by karyotyping (Zhang et al., 2011). Similarly, the study by Han et al, which examined 31,615 high-risk pregnancies, reported 36.9\% was trisomy 21 (Han et al., 2008). Our study results confirmed that trisomy 21 was the most frequent chromosomal aberration in pregnancy.

The most common structural aberration was inversion (7.3\%), followed by reciprocal (5.3\%), and deletion (4.5\%). 
The majority of those disorders found with the history of miscarriage and/or abortion and abnormal ultrasound findings. This pattern was consistent with a study by Ocak et al in 2013 (Ocak et al., 2014). Reciprocal translocations from parental carriers increase the risk of miscarriage and implantation failure. Genetic material unbalance causes congenital malformations, intra-uterine growth restriction which can be detected via ultrasound. In such condition, preimplantation genetic diagnosis (PGD) was appropriate. Studies have shown that clinical pregnancy rate per oocyte increased from $29.0 \%$ to $38.0 \%$ and the rate of miscarriage decreased from $92.0 \%$ to $12.5 \%$ (Dietterich, Check, Choe, Nazari, \& Lurie, 2002). Moreover, PGD could be indicated for couples suspected with genetic carriers aiming at enhanced genetic quality and avoiding spreading mutations in the population.

In this study, 54 cases of polymorphism were detected (7.0\%). Among those, 8 cases with increased nuchal translucency and 8 cases of abnormal ultrasound, other cases were positive with maternal serum screening test. Polymorphisms were possibly STR, SNP or repeat of the satellite. However, there was no reason for inherited conditions. A recent study raised the hypothesis which chromosomal polymorphism at heterochromatin was responsible for decreasing reproductive function and recurrent miscarriage among infertile couples (Madon, Athalye, \& Parikh, 2005).

\section{Conclusion}

Our study indicated that the overall rate of chromosomal disorders, in which autosomal chromosome disorders and structural rearrangements were the most frequently found among chromosomes abnormal cases. Regarding amniocentesis indication, abnormal ultrasound finding was the most common reason for amniocentesis test. This study provides reliable evidence to develop prenatal counseling and pregnancy management.

\section{Acknowledgments}

We would like to thank the participants for their cooperation and the medical staffs from the Center for Prenatal Diagnosis at the National Hospital of Obstetrics and Gynecology who gave valuable support to this study.

\section{Data Availability}

The data could be obtained by contacting corresponding author.

\section{Funding Statement}

The authors declared that no grants were involved in supporting this work.

\section{Competing Interests Statement}

No competing interests were disclosed.

\section{References}

Aminu, M., Unkels, R., Mdegela, M., Utz, B., Adaji, S., \& van den Broek, N. (2014). Causes of and factors associated with stillbirth in low- and middle-income countries: a systematic literature review. BJOG: An International Journal of Obstetrics \& Gynaecology, 121, 141-153. https://doi.org/10.1111/1471-0528.12995

Caron, L., Tihy, F., \& Dallaire, L. (1999). Frequencies of chromosomal abnormalities at amniocentesis: over 20 years of cytogenetic analyses in one laboratory. Am J Med Genet, 82(2), 149-154. https://doi.org/10.1002/(SICI)1096-8628(19990115)82:2<149::AID-AJMG10>3.0.CO;2-L

Chang, Y. W., Chang, C. M., Sung, P. L., Yang, M. J., Li, W. H., Li, H. Y., .. W Wang, P. H. (2012). An overview of a 30-year experience with amniocentesis in a single tertiary medical center in Taiwan. Taiwan J Obstet Gynecol, 51(2), 206-211. https://doi.org/10.1016/j.tjog.2012.04.007

Dietterich, C., Check, J. H., Choe, J. K., Nazari, A., \& Lurie, D. (2002). Increased endometrial thickness on the day of human chorionic gonadotropin injection does not adversely affect pregnancy or implantation rates following in vitro fertilization-embryo transfer. Fertil Steril, 77(4), 781-786. https://doi.org/ 10.1016/S0015-0282(01)03276-9

Ferguson-Smith, M. A., \& Yates, J. R. (1984). Maternal age specific rates for chromosome aberrations and factors influencing them: report of a collaborative european study on 52965 amniocenteses. Prenat Diagn, 4 Spec No, 5-44. https://doi.org/10.1002/pd.1970040704

Field, B., \& Kerr, C. (1988). Reproductive behaviour and consistent patterns of abnormality in offspring of Vietnam veterans. J Med Genet, 25(12), 819-826. https://doi.org/10.1136/jmg.26.9.602

Han, S. H., An, J. W., Jeong, G. Y., Yoon, H. R., Lee, A., Yang, Y. H., . . . Lee, K. R. (2008). Clinical and cytogenetic findings on 31,615 mid-trimester amniocenteses. Korean J Lab Med, 28(5), 378-385. https://doi.org/10.3343/kjlm.2008.28.5.378 
Hassold, T., \& Hunt, P. (2009). Maternal age and chromosomally abnormal pregnancies: what we know and what we wish we knew. Curr Opin Pediatr, 21(6), 703-708. https://doi.org/10.1097/MOP.0b013e328332c6ab

Hook, E. B. (1983). Chromosome abnormalities and spontaneous fetal death following amniocentesis: further data and associations with maternal age. Am $J$ Hum Genet, 35(1), 110-116. https://doi.org/10.1097/00006254-198311000-00014

Hook, E. B., Cross, P. K., \& Schreinemachers, D. M. (1983). Chromosomal abnormality rates at amniocentesis and in live-born infants. Jama, 249(15), 2034-2038. 10.1001/jama. https://doi.org/1983.03330390038028

Jacobson, C. B., \& Barter, R. H. (1967). Intrauterine diagnosis and management of genetic defects. Am J Obstet Gynecol, 99(6), 796-807. https://doi.org/10.1016/0002-9378(67)90395-X

Madon, P. F., Athalye, A. S., \& Parikh, F. R. (2005). Polymorphic variants on chromosomes probably play a significant role in infertility. Reprod Biomed Online, 11(6), 726-732. https://doi.org/10.1016/S1472-6483(10)61691-4

Ocak, Z., Ozlu, T., Yazicioglu, H. F., Ozyurt, O., \& Aygun, M. (2014). Clinical and cytogenetic results of a large series of amniocentesis cases from Turkey: report of 6124 cases. J Obstet Gynaecol Res, 40(1), 139-146. https://doi.org/10.1111/jog.12144

Özer, L., Ünsal, E., Ayvaz, Ö., Şen, A., \& Baltac1, V. (2016). Prenatal Cytogenetic Findings in 13.466 Cases of High-Risk Pregnant Women in One Laboratory. Gynecol Obstet Reprod Med, 19(3).

Pham, V., Nguyen, H., Tho le, H., Minh, T. T., Lerdboon, P., Riel, R., . . Kaljee, L. M. (2012). Evaluation of three adolescent sexual health programs in ha noi and khanh hoa province, Vietnam. AIDS Res Treat, 2012, 986978. https://doi.org/10.1155/2012/986978

Seabright, M. (1971). A rapid banding technique for human chromosomes. Lancet, 2(7731), 971-972.

Tseng, J. J., Chou, M. M., Lo, F. C., Lai, H. Y., Chen, M. H., \& Ho, E. S. (2006). Detection of chromosome aberrations in the second trimester using genetic amniocentesis: experience during 1995-2004. Taiwan $J$ Obstet Gynecol, 45(1), 39-41. https://doi.org/10.1016/s1028-4559(09)60188-1

Wang, P. H., \& Cheng, M. H. (2009). Amniotic fluid cytokines predict pregnancy outcome: myth or reality? J Chin Med Assoc, 72(12), 617-618. https://doi.org/10.1016/s1726-4901(09)70441-1

Zhang, Y. P., Wu, J. P., Li, X. T., Lei, C. X., Xu, J. Z., \& Yin, M. (2011). [Karyotype analysis of amniotic fluid cells and comparison of chromosomal abnormality rate during second trimester]. Zhonghua Fu Chan Ke Za Zhi, 46(9), 644-648.

\section{Copyrights}

Copyright for this article is retained by the author(s), with first publication rights granted to the journal.

This is an open-access article distributed under the terms and conditions of the Creative Commons Attribution license (http://creativecommons.org/licenses/by/4.0/). 